Enhancement of Redox Cycl ing Currents at I nt er di gi t at ed El ectrodes wi th El evat ed Fi ngers

\begin{tabular}{|l|l|}
\hline 著者 & $\begin{array}{l}\text { Nor i t a Nasao, Hayashi Kat suy oshi, Hor i uchi } \\
\text { Tsut om, Shi bano Sayaka, Yamant o Kat sunobu, } \\
\text { Aoki Koi chi }\end{array}$ \\
\hline $\begin{array}{l}\text { j our nal or } \\
\text { publ i cat i on ti tl e }\end{array}$ & Jour nal of The El ect r ochemi cal Soci et y \\
\hline vol une & 161 \\
\hline number & 4 \\
\hline page range & H178 H182 \\
\hline year & 2014 10 \\
\hline URL & ht t p: //hdl . handl e. net /10098/8598 \\
\hline
\end{tabular}




\title{
Enhancement of Redox Cycling Currents at Interdigitated Electrodes with Elevated Fingers
}

\author{
Masao Morita, ${ }^{a, e, z}$ Katsuyoshi Hayashi,b Tsutomu Horiuchi, ${ }^{\text {b }}$ Sayaka Shibano, ${ }^{c}$

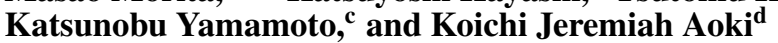

${ }^{a}$ NTT Advanced Technology Corporation, Atsugi, Kanagawa 243-0124, Japan

${ }^{b}$ NTT Microsystem Integration Laboratories, Kanagawa 243-0198, Japan

${ }^{c}$ BAS Inc., 1-28-12 Mukojima, Sumida, Tokyo 131-0033, Japan

${ }^{d}$ Department of Applied Physics, University of Fukui, Fukui 910-0017, Japan

\begin{abstract}
An ideal interdigitated array (IDA) electrode is composed of a pair of comb-type finger electrodes flush with an insulating wall. However, the fingers that compose an actual IDA are elevated from the gap plane by their thickness. The side wall of the elevated part can work as an active electrode, and increases the current for redox cycling reactions, as in a thin layer cell. Here, the elevated fingers are deliberately fabricated to increase the redox cycling currents. Our fabrication technique involves combining the carbonization of thick polyimide films with photolithography on quartz substrates. One type of fabricated electrode has an elevated height ranging from 0.01 to $4.5 \mu \mathrm{m}$ for a $5 \mu \mathrm{m}$ gap. The other type has a height of $0.05 \mu \mathrm{m}$ for gaps ranging from 0.25 to $2 \mu \mathrm{m}$. The theoretical current is evaluated by solving numerically the Laplace equation for the redox concentration by using the finite element method. An approximate equation for diffusion controlled currents is obtained based on the concept that the current is the sum of the current at the flat IDA and that at the area of the electrode side wall. Voltammograms of ferrocene derivatives demonstrate the validity of the approximate equation for the enhancement of the current.

(C) 2014 The Electrochemical Society. [DOI: 10.1149/2.101403jes] All rights reserved.
\end{abstract}

Manuscript submitted December 12, 2013; revised manuscript received January 8, 2014. Published January 28, 2014.

Interdigitated array (IDA) electrodes are composed of two addressable interdigitated comb-like electrodes. ${ }^{1,2}$ They have been used as sensitive electrochemical sensors that employ steady state redox cycling reactions, ${ }^{3-6}$ as sensors in flowing systems, ${ }^{7-11}$ to measure the conductivity of films, ${ }^{12-18}$ as electrochemical detectors of redox interactions in films, ${ }^{19-24}$ and as tools for detecting reaction intermediates. ${ }^{25-27}$ Their advantage lies in the establishment of amplified steady-state currents by employing an electrochemical control at the generator and the detector electrode. The steady state is caused by redox cycling, by which the reduced species generated at the cathode comb is transferred to the anode, where it is oxidized. In other words, one species moves back and forth between two neighboring finger electrodes of different comb electrodes. ${ }^{5,28,29}$ A larger current is obtained per finger as the distance between the two finger electrodes decreases. ${ }^{3,28,29}$ Even if only the reduced species is initially present in solution, the oxidation current can flow with the help of a counter electrode to generate the oxidized species. ${ }^{28,30}$ If either the reduced or oxidized species is unstable, redox cycling is observed as the result of reactions with auxiliary redox couples. ${ }^{3,31-33}$ It is not necessary to use a bi-potentiostat to observe redox cycling voltammograms if the potential at one comb is controlled by sacrificial electrodes. ${ }^{34-36}$

IDA fabrication techniques have concentrated on miniaturization $^{3,30}$ and electrode materials. ${ }^{37-40}$ The aim of the former approach is to reduce the time needed to attain steady-state voltammograms or have the correction efficiency reach unity. According to the theory of diffusion controlled current at an IDA, ${ }^{28}$ current with an average finger distance, $w$, is approximately proportional to $\ln (2.55 \mathrm{w} / \mathrm{g})$, where $g$ is the width of the gap between neighboring fingers. The ratio, $w / g$, is conventionally two owing to the limitation imposed by fabrication techniques. Since it is not practical to reduce only $g$, while maintaining $w$, the current does not vary greatly with changes in $g$ and $w$.

The model of the redox cycling at the IDA assumes that the fingers of the IDA are mounted flush with the gap plane. ${ }^{28}$ Since IDAs are fabricated by depositing electrode materials through a lithographical pattern, the fingers are necessarily raised above the gap plane. This elevation increases the currents caused by the redox cycling between neighboring elevated parts, ${ }^{32,41-44}$ such as the redox cycling currents in a thin layer cell. ${ }^{4-47}$ The elevation height can be increased only by the deposition of electrode materials over a long period. Unfortunately, no

${ }^{\text {e}}$ Present address: Graduate School of Science, Tohoku University, Aramaki, Aoba, Sendai, Miyagi 980-8578, Japan.

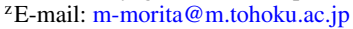

quantitative relationship has yet been reported between the elevation and the currents, only the specific geometry of the IDAs. ${ }^{43,44}$ This work is directed toward obtaining a guideline for enhancing the current by varying the elevation. Our goal is to obtain the quantitative relationship between the currents and the geometry of the elevated IDA by solving the steady-state diffusion equation numerically. The relationship is verified experimentally for various elevated IDAs.

\section{Theory}

The anode and cathode of our IDA with elevated fingers have the same width, as illustrated in Fig. 1. We designed a unit cell to consist of a two-dimensional domain enclosed by a neighboring anode and cathode, their gap, and their centerlines. Figure 2 shows a unit cell model and the coordinates. The concentration, $c$, of the reduced species is subject to two-dimensional diffusion in a steady state,

$$
\partial^{2} c / \partial x^{2}+\partial^{2} c / \partial y^{2}=0
$$

The boundary conditions are

$$
\begin{aligned}
& c=0 \text { at the anode }(x=g / 2,0<y<h ; g / 2<x<w / 2, y=h) \\
& c=c^{*} \text { at the cathode } \\
& \quad(x=-g / 2,0<y<h ;-w / 2<x<-g / 2, y=h) \\
& \partial c / \partial y=0 \text { at the gap }(y=0,|x|<g / 2) \\
& \partial c / \partial x=0 \text { at the unit boundaries }(x= \pm w / 2, y>h)
\end{aligned}
$$

where $c^{*}$ is the bulk concentration of the reduced species. We solve the above boundary value problem for the Laplace equation by using the finite element method with the auxiliary condition, $\partial c / \partial y=0$ at the top of the unit cell $(|x|<w / 2, y=H)$, where $H$ is the height of the unit cell so that it does not alter the calculated concentration profiles. The finite element method software was XFEM113 (freeware). The mesh was generated with voro95 (freeware).

Small meshes were adopted near the electrodes to express large concentration variations accurately. We used about 4000 elements. The height of the cell, $H$, was considered to be roughly twice as large as $h$ or $w$ so it had negligible effect on the concentration profiles. The concentration normalized by $c^{*}$ is a function of two geometrical parameters, $g / w$ and $h / w$. Figure 2 shows an example of concentration profiles obtained by employing color gradation for two geometries at $h / w=$ (A) 0.4 and (B) 0 . The profiles are independent of the diffusion 


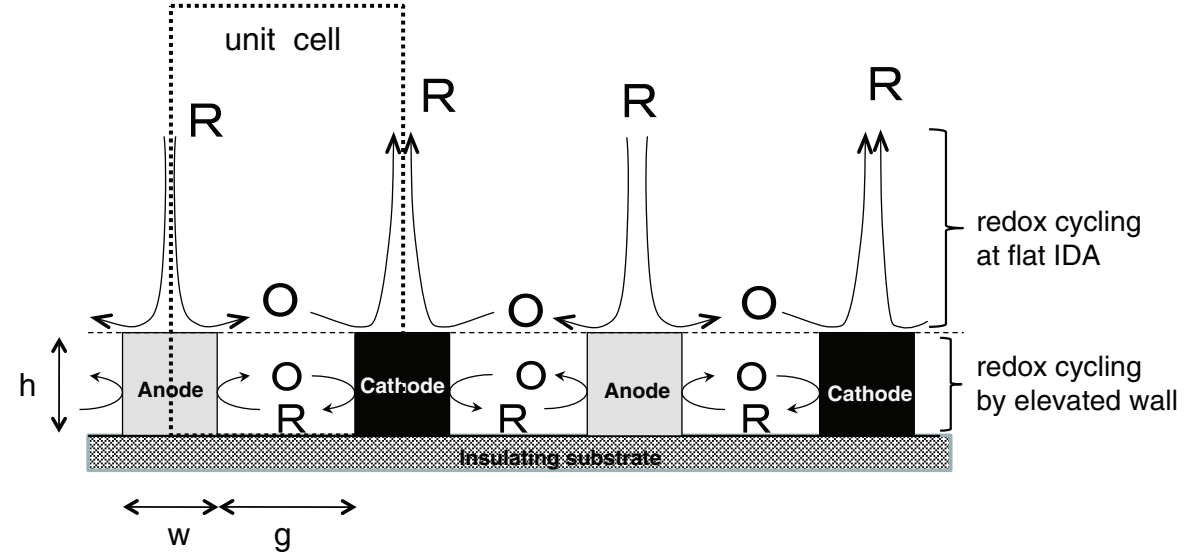

Figure 1. Illustration of mass transport of redox species at an elevated IDA when the reduced species is initially present in the bulk.
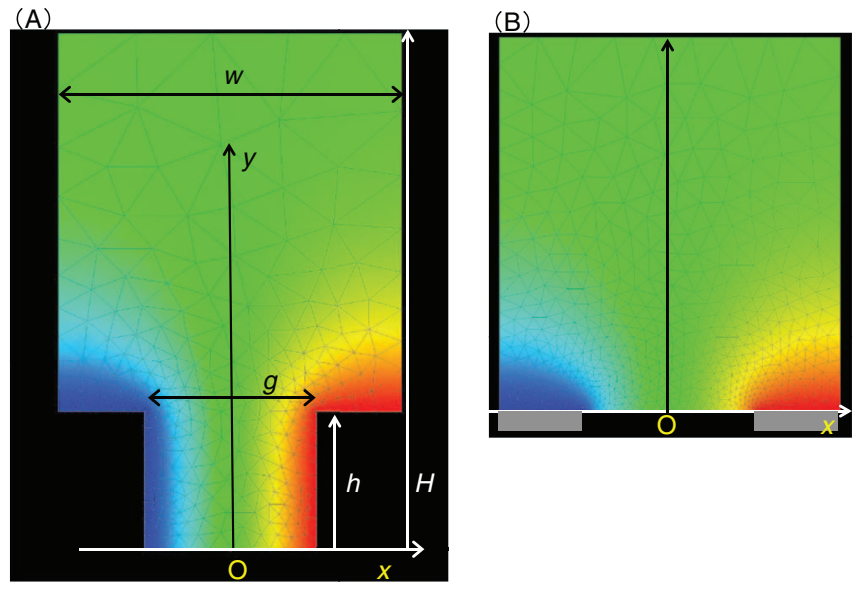

Figure 2. Concentration distributions in a unit cell at $g / w=0.5$, (A) $h / w$ $=0.4$ and (B) $h / w=0$, obtained with the finite element method for around 900 meshes.

coefficient because it is canceled out in Eqs. 1 and 2. The domains near the electrode have identical colors because the concentration is controlled by the electrode potential through the Nernst equation. The color variation in the domain, $|x|<g / 2$ and $0<y<h$ indicates that the area of the electrode side wall participates in the electrolysis, as in a thin layer cell. The color variation on the line $y=h$ in (A) is slightly smoother than that on $y=0$ in (B), because the electrolysis at the area of the electrode side wall generates a linear concentration profile. To clarify the concentration variation, the concentration profiles on these lines are plotted in Fig. 3. Profile (c) at the gap of the flat IDA

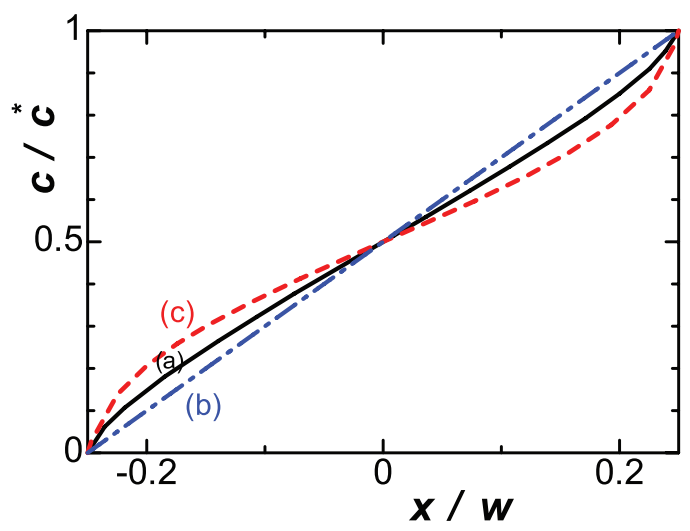

Figure 3. Concentration profiles of the model in Fig. 2A on lines (a) at $y=h / w$ and (b) at $y=0$, and (c) the model in Fig. 2B on a line at $y=0$.
(Fig. 2B) shows large concentration variations near the electrode edge $(x / w= \pm 0.25)$ owing to diffusion in the direction of the $x$-axis. In contrast, profile (b) at the gap at the IDA with $h / w=0.4$ is a line that is the same as in the thin layer cell because the square domain of $\mathrm{h} / \mathrm{g}$ in Fig. 2 corresponds to the thin layer cell. Profile (a) on the line at $y=h / w$ is between the above two profiles. It is this intermediate profile that makes the current complicated.

The diffusion controlled limiting current, $I_{\text {fng }}$, per finger electrode of length $b$ is obtained through

$$
\frac{I_{\text {fng }}}{b F}=2 D \int_{0}^{h}\left(\frac{\partial c}{\partial x}\right)_{x=g / 2} \mathrm{~d} y+2 D \int_{0}^{(w-g) / 2}\left(\frac{\partial c}{\partial y}\right)_{y=h} \mathrm{~d} x
$$

where $D$ is the diffusion coefficient, which is common to both the reduced and oxidized species. If the value of the diffusion coefficient of the reduced species is different from that of the oxidized species, the boundary conditions of $c=0$ and $c=c^{*}$ become only approximations even in the potential domain of a limiting current. The flux at the electrode was calculated by taking account of the gradient of the output concentrations at elements including the electrode surface. The software for the flux was homemade. After carrying out the integration in Eq. 3 numerically, we obtained the dimensionless current per finger, $I_{\mathrm{fng}} / b F c^{*} D$. The current density values for $h=0$, which should be identical to those at the conventional IDA, were 6-8\% smaller than the analytical expression for the IDA current. ${ }^{28}$ This is probably because the numerical calculation fails to evaluate the infinite current density at the edge electrode. The variations in $I_{\text {fng }} / b F c^{*} D$ with $h / w$ are shown in Fig. 4 for several $g / w$ values. The current increases linearly with increases in $h$. This agrees with Fig. 14 in Ref. 44. The linearly increasing part is predicted to be proportional to $h / g$ by analogy with the current in a thin layer cell. We plotted the slope against

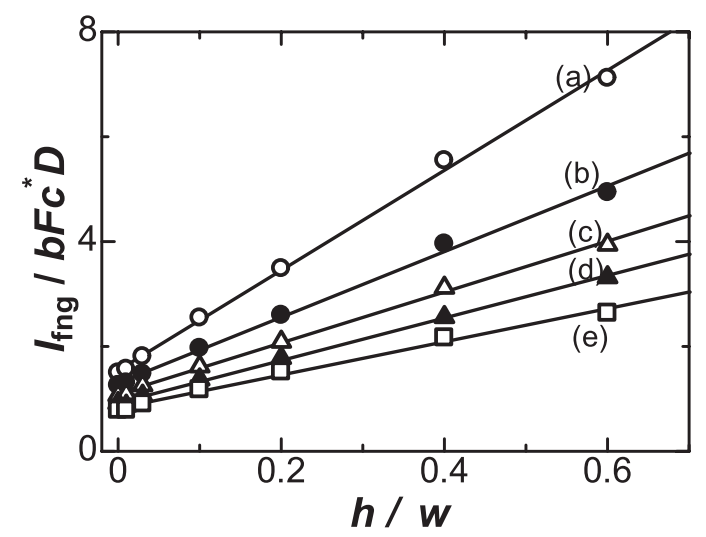

Figure 4. Dependence of the dimensionless current at a finger electrode on $h$ for $g / w=$ (a) 0.2 , (b) 0.3 , (c) 0.4 , (d) 0.5 and (e) 0.6 , calculated by the finite element method. 


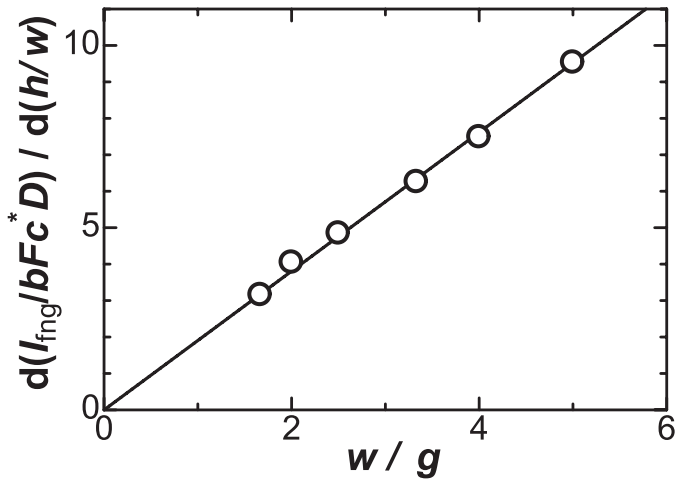

Figure 5. Plot of the slope of the variations in Fig. 4 against 1/g.

$w / g$ in Fig. 5 to show the proportionality. Therefore, the area of the electrode side wall works as an electrode, as in a thin layer cell with the width $g$. The proportionality in Fig. 5 is empirically formulated as $\left(I_{\text {fng, }} / b F c^{*} D\right)(g / w)=1.90$, or

$$
I_{\text {fng,h }}=1.90 b F c^{*} D h / g
$$

where $I_{\text {fng,h }}$ is the contribution to the area of the electrode side wall. Since the finger electrode has two areas of the electrode side wall, the current per protrusion is $0.95 b F c^{*} \mathrm{Dh} / \mathrm{g}$. The coefficient of 0.95 instead of unity is obviously the current for the thin layer cell. The $5 \%$ loss may be ascribed to both an underestimation of the numerical computation by the finite difference in Eq. 3 and the complicated concentration distributions in Fig. 3.

Equation 3 is composed of the sum of the currents at the elevated IDA and at the flat IDA. Since the first term is close to the value (Eq. 4) of the thin layer cell, the current at the IDA with the elevation may be expressed numerically by the sum of $I_{\mathrm{fng}, \mathrm{h}}$ and the flat IDA current. By using the approximate equation for the current at the flat IDA, ${ }^{28}$ we can express Eq. 3 as

$$
\frac{I_{\text {fng }}}{b F c^{*} D}=1.90 \frac{h}{g}+0.637 \ln \left(2.55 \frac{w}{g}\right)-0.19\left(\frac{g}{w}\right)^{2}
$$

The sum of the second and third terms in Eq. 5, expressing the contribution of the flat IDA, is close to 1.0 for $0.2<g / w<0.55$ within $5 \%$ error. Therefore, Eq. 5 can be approximated as

$$
I_{\text {fng }}=b F c^{*} D(1.90 h / g+1)
$$

This equation indicates that a fabrication technique that makes the electrode thick will enhance the current.

\section{Experimental}

Fabrication of elevated IDA.- We fabricated three types of carbon films whose thicknesses, $h$, were of the order of $0.05,1$, and $5 \mu \mathrm{m}$. The carbon film was formed on a quartz substrate via the carbonization of polyimide film. Polyamic acid varnish (Pyre-ML, du Pont, USA) was spin-coated on the substrate and baked at $250^{\circ} \mathrm{C}$ for 2 hours. The film thickness was controlled by adjusting the spin-coating speed and varnish concentration. After carbonizing the polyimide film on the substrate at $1000^{\circ} \mathrm{C}$ for 2 hours in a vacuum, we obtained carbon films 1.3 and $4.5 \mu \mathrm{m}$ thick. The film thickness was obtained with a probe type step profiler, Dektak3030 (ULVAC, Inc.). The conductivity of the $4.5 \mu \mathrm{m}$ thick carbon film was about $100 \mathrm{ohm} \mathrm{cm}^{-2}$. The carbon films with a thickness of around $0.05 \mu \mathrm{m}$ were formed on a quartz substrate as described elsewhere. ${ }^{48,49}$

The substrates coated with conducting carbon films were fabricated into IDA electrodes by lithography. ${ }^{39,48,49}$ A silicon-based positive photoresist (FH-SP, NTT-AT, Japan) was used for resist patterning because it could endure the subsequent oxygen dry etching process that removed the thick carbon film that was not covered with resist.

\begin{tabular}{|c|c|c|c|c|c|c|}
\hline IDA & $w / \mu \mathrm{m}$ & $g / \mu \mathrm{m}$ & $h / \mu \mathrm{m}$ & $b / \mathrm{mm}$ & $m$ & $I_{\mathrm{L}} / \mu \mathrm{A}$ \\
\hline 1 & 15.0 & 5.1 & 0.01 & 2 & 65 & 8.5 \\
\hline 2 & 15.0 & 4.7 & 1.30 & 2 & 65 & 14.2 \\
\hline 3 & 15.0 & 5.1 & 4.50 & 2 & 65 & 21.9 \\
\hline 4 & 4.0 & 2.0 & 0.05 & 1 & 125 & 0.7 \\
\hline 5 & 2.0 & 1.0 & 0.05 & 1 & 250 & 1.6 \\
\hline 6 & 1.0 & 0.5 & 0.05 & 1 & 500 & 3.5 \\
\hline 7 & 0.9 & 0.45 & 0.05 & 1 & 555 & 3.7 \\
\hline 8 & 0.8 & 0.4 & 0.05 & 1 & 625 & 4.2 \\
\hline 9 & 0.7 & 0.35 & 0.05 & 1 & 714 & 4.9 \\
\hline 10 & 0.6 & 0.3 & 0.05 & 1 & 833 & 6.0 \\
\hline 11 & 0.5 & 0.25 & 0.05 & 1 & 1000 & 7.5 \\
\hline
\end{tabular}

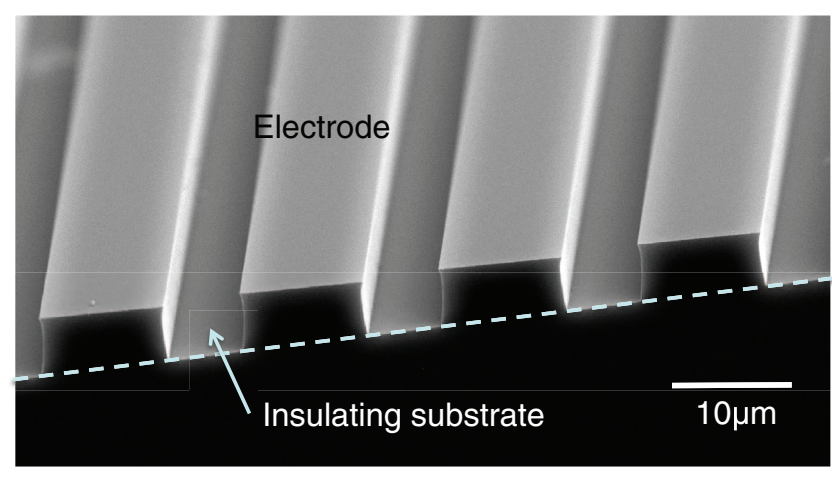

Figure 6. SEM image of a cross section of IDA3.

$m$ : number of fingers per comb

$I_{\mathrm{L}}$ : average of four limiting currents

The resist film was used to cover the electric leads on the carbon film, and was heated at $200^{\circ} \mathrm{C}$ to yield a hard film.

Figure 6 shows a SEM image of a cross section of IDA3, where the cross section was exposed by means of RealSurfaceView VE7800 (KEYENCE). The cross sectional geometry is $w=15 \mu \mathrm{m}$ $h=4.5 \mu \mathrm{m}$ and $g=4.5 \mu \mathrm{m}$. The geometric features of all the IDA electrodes are listed in Table I.

Chemicals and apparatus. - We used (ferrocenylmethyl)trimethyl ammonium bromide (FcTMA) (Tokyo Kasei, Japan) and phosphate buffered saline (PBS) (Gibco, USA) as a redox species and supporting electrolyte, respectively, without purification. Electrochemical measurements were performed with a dual potentiostat, ALS/CHI model 1000 (CHI Instruments Inc., USA). A $\mathrm{Pt}$ wire and a silver/silver chloride $(\mathrm{Ag} / \mathrm{AgCl}, \quad[\mathrm{NaCl}]$ $=3 \mathrm{M}$ ) electrode were used as a counter and a reference electrode, respectively.

\section{Results and Discussion}

When a scanned potential is applied commonly to the two combs of a flat IDA in a solution including redox species, the voltammogram should be similar to a linear sweep voltammogram at a planar electrode with the same area as the two combs and gaps, according to the concept of partially blocked electrodes ${ }^{50}$ Figure 7 shows voltammograms obtained at the flat IDA(a). The appearance of a pair of peaks suggests control of linear diffusion. A diffusion layer at a finger overlaps those at neighboring fingers when there is a lapse in the electrolysis. Then the overall diffusion layer should be close to the layer generated by the planar electrode. This explains the appearance of the peak. The voltammogram was close to that calculated theoretically by linear diffusion (c). Although the elevated IDAs have net electrode surface areas larger than the projected area, the peak currents were 


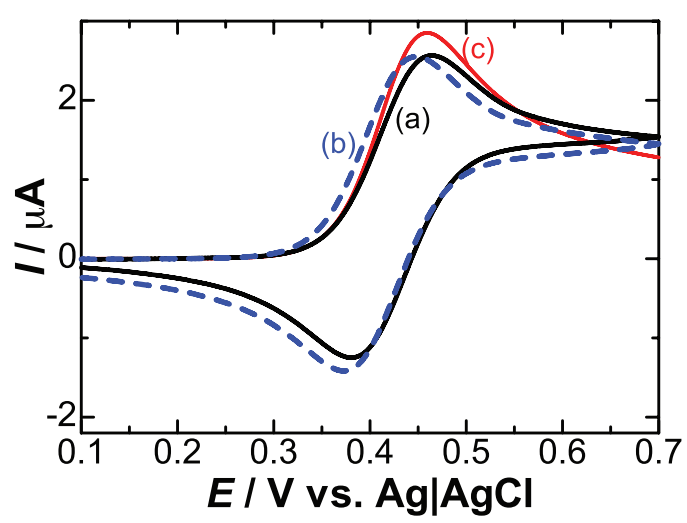

Figure 7. Cyclic voltammograms in $1 \mathrm{mM}$ FcTMA solution at $v=10 \mathrm{mV}$ $\mathrm{s}^{-1}$ at (a) IDA1 and (b) IDA2 when a common scan voltage was applied to the two combs; and (c) linear sweep voltammogram calculated for the same conditions as used for the experiments at the planar electrode.

almost independent of the $h$ values (in Fig. 7b). This independence is supported by the concept of partially blocked electrodes.

One comb was set at a constant potential of $0 \mathrm{~V}$, whereas the potential was scanned at the other electrode in a solution containing FcTMA, called a dual mode. When the current at the former comb was plotted against the scanned potential, steady-state voltammograms were obtained for three values of $h$, as shown in Fig. 8. The limiting current increased with increases in $h$. The collection efficiency, defined by the ratio of the limiting current at the comb for a scanned potential to that for the constant potential, ranged from 0.92 to 0.96 .

The steady-state voltammograms obtained at the flat IDA in the limiting current domain. e.g. $E>0.6 \mathrm{~V}$ in Fig. 8 did not vary greatly with the gap width, which ranged from 0.25 to $20 \mu \mathrm{m}$. This invariance agrees with the theoretical prediction, which indicates that the sum of the second and third terms in Eq. 5 is close to unity for $0.2<\mathrm{g} / \mathrm{w}$ $<0.55$. The logarithmic variation with $g$ is characteristic of the solution of the two-dimensional Laplace equation. ${ }^{51,52}$

Background corrected limiting currents, $I_{\mathrm{L}}$, at $E>0.6 \mathrm{~V}$ in Fig. 8 were determined by averaging four current voltage curves at the forward scan and the backward scan at the two combs. These currents are listed in Table I. The $I_{\text {fng }}$ values were obtained from the average current and the number of fingers. Since Eq. 5 predicts that $I_{\text {fng }}$ is proportional to $h / g$ for any $g$ value, we plotted the variations in $I_{\text {fng }} / b$ with $h / g$ for two types of IDAs with $w=15 \mu \mathrm{m}$ and $0.5 \mu \mathrm{m}<w<$ $4 \mu \mathrm{m}$, as shown in Fig. 9. These values are close to the line calculated from Eq. 5 for $c^{*}=1 \mathrm{mM}$ and $D=0.63 \times 10^{-5} \mathrm{~cm}^{2} \mathrm{~s}^{-1}$ of FcTMA

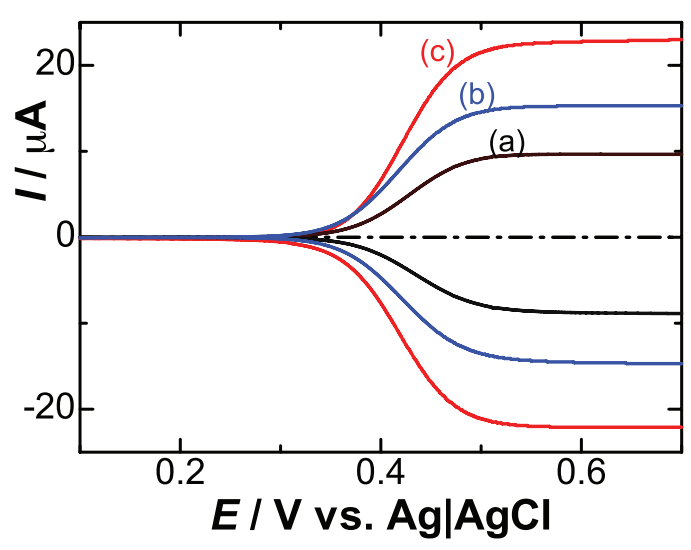

Figure 8. Voltammograms of $1 \mathrm{mM}$ FcTMA at IDAs for $h=($ a) $0.01 \mu \mathrm{m}$, (b) $1.3 \mu \mathrm{m}$ and (c) $4.5 \mu \mathrm{m}$ when the potential at one comb was scanned for $10 \mathrm{mV} \mathrm{s}^{-1}$ while $0.0 \mathrm{~V}$ was applied to the other comb. The negative currents correspond to a comb at a constant potential of $0.0 \mathrm{~V}$.

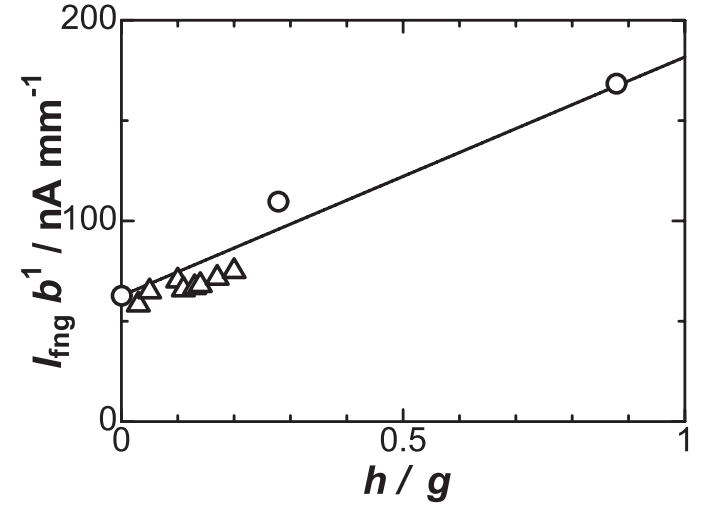

Figure 9. Variation in the redox cycling currents per finger with the elevated height at (circles) IDA1-3 and (triangles) IDA4-11. The line was calculated from Eq. 5. The errors in the triangles roughly correspond to the size of the marks.

where the value of the diffusion coefficient was obtained in $0.5 \mathrm{M}$ $\mathrm{KCl}$ aqueous solution by using the combination of a regular electrode and a microelectrode". ${ }^{53}$ The $I_{\text {fng }} / b$ values at the IDAs of $w<4 \mu \mathrm{m}$ (triangles) were smaller than the theoretical values, probably because not all the fingers work as active electrodes owing to defects in the electric connections over all the fingers.

\section{Conclusions}

The elevated IDA enhances the steady-state redox cycling currents. The enhancement is linearly related to the elevated height. The limiting current can be approximated as the sum of the current at the flat electrode and that at the facing elevated parts similar to the behavior observed in a thin layer cell. The latter component is, however, 95\% of the current in the thin layer cell with the distance $g$ and the area $b h$. The approximation is not explicitly a function of $w$. Therefore, a reduction in the size of the unit cell does not enhance the current per finger. Cyclic voltammograms at a potential common to the two combs are almost the same as those at a planar electrode with the same projected area at the IDA, independent of $h$.

\section{Acknowledgments}

We thank Dr. Kazuo Imai and Hisao Tabei for helpful discussions and suggestions regarding the fabrication of the thick IDA electrodes.

\section{References}

1. D. G. Sanderson and L. B. Anderson, Anal. Chem., 57, 2388 (1985).

2. C. E. Chidsey, B. J. Feldman, C. Lundgren, and R. W. Murray, Anal. Chem., 58, 601 (1986).

3. O. Niwa, M. Morita, and H. Tabei, Electroanalysis, 3, 163 (1991).

4. C. S. Cha, M. J. Shao, and C. C. Liu, Sens. Actuat. B, 2, 239 (1990).

5. O. Niwa, Y. Xu, H. H. Brian, and W. R. Heineman, Anal. Chem., 65, 1559 (1993).

6. J. H. Thomas, S. K. Kim, P. J. Hesketh, H. B. Halsall, and W. R. Heineman, Anal. Biochem., 328, 113 (2004).

7. E. D. Goluch, B. Wolfrum, P. S. Singh, M. A. G. Zevenbergen, and S. G. Lemay, Anal. Bioanal. Chem., 394, 447 (2009).

8. D. Daniel and I. G. R. Gutz, Talanta, 68, 429 (2005).

9. R. Kurita, H. Tabei, Z. Liu, T. Horiuchi, and O. Niwa, Sens. Actuat. B, 71, 82 (2000).

10. C. Belmont and H. H. Girault, J. Appl. Electrochem., 24, 719 (1994).

11. T. Y. Ou, S. Moldoveanu, and J. L. Anderson, J. Electroanal. Chem., 247, 1 (1988).

12. G. Casalbore-Miceli, A. Zanelli, E. M. Girotto, A. W. Rinaldi, A. F. Rubira, and A. Berlin, Electrochim. Acta, 51, 5268 (2006).

13. M. Krondak, G. Broncová, S. Anikin, A. Merz, and V. M. Mirsky, J. Solid State Electrochem., 10, 185 (2006).

14. I. A. Greene, F. Wu, J. Z. Zhang, and S. Chen, J. Phys. Chem. B, 107, 5733 (2003).

15. M. Mohamedi, S.-J. Lee, D. Takahashi, M. Nishizawa, T. Itoh, and I. Uchida, Electrochim. Acta, 46, 1161 (2001).

16. M. K. Ram, M. Adami, M. Sartore, M. Salerno, S. Paddeu, and C. Nicolini, Synth. Met., 100, 249 (1999).

17. H. Okamoto, Y. Ando, and T. Kotaka, Synth. Met., 96, 7 (1998). 
18. M. Shibuya, T. Nishina, T. Matsue, and I. Uchida, J. Electrochem. Soc., 143, 3157 (1996).

19. H. Yamaguchi and K. Matsuda, Chem. Lett., 38, 946 (2009).

20. J. P. Collman, A. Hosseini, T. A. Eberspacher, and C. E. D. Chidsey, Langmuir, 25, 6517 (2009).

21. N. K. Devaraj, P. H. Dinolfo, C. E. D. Chidsey, and J. P. Collman, J. Am. Chem. Soc., 128, 1794 (2006)

22. B. A. Gregg and R. A. Cormier, J. Phys. Chem. B, 102, 9952 (1998)

23. C. Chen, T. A. Postlethwaite, J. E. Hutchison, E. T. Samulski, and R. W. Murray, J. Phys. Chem., 99, 8804 (1995).

24. A. Aoki and A. Heller, J. Phys. Chem., 97, 11014 (1993).

25. C.-G. Lee, H. Ojima, and M. Umeda, Electrochim. Acta, 53, 3029 (2008).

26. K. Hayashi, Y. Iwasaki, T. Horiuchi, K Sunagawa, and A. Tate, Anal. Chem., 77, $5236(2005)$.

27. C. S. Cha, M. J. Shao, and C. C. Liu, Sens. Actuat. B, 2, 239 (1990).

28. K. Aoki, M. Morita, O. Niwa, and H. Tabei, J. Electroanal. Chem., 256, 269 (1988)

29. O. Niwa, M. Morita, and H. Tabei, Anal. Chem., 62, 447 (1990).

30. K. Hayashi, J. Takahashi, T. Horiuchi, Y. Iwasaki, and T. Haga, J. Electrochem. Soc., 155, J240 (2008).

31. M.-C. Chuang, H.-Y. Lai, J. A. Ho, and Y. Y. Chen, Biosens. Bioelect., 41, 602 (2013).

32. V. A. T. Dam, W. Olthuis, and A. v. d. Berg, Analyst, 132, 365 (2007).

33. Z. M. Liu, T. Y. You, X. R. Yang, and E. K. Wang, Electroanalysis, 11, 53 (1999).

34. T. Horiuchi, O. Niwa, and H. Tabei, Anal. Chem., 66, 1224 (1994).

35. T. Horiuchi, O. Niwa, M. Morita, and H. Tabei, Anal. Chem., 64, 3206 (1992).

36. H. Tabei, T. Horiuchi, O. Niwa, and M. Morita, J. Electroanal. Chem., 326, 339 (1992).
37. W. Zhang, S. Xie, M. Li, H. Chen, L. Ma, J. Jian, and J. Coll. Czech. Chem. Comm., 74, 393 (2009).

38. G. C. Fiaccabrino, X.-M. Tang, N. Skinner, N. F. De Rooij, and M. Koudelka-Hep, Anal. Chim. Acta, 326, 155 (1996).

39. O. Niwa and H. Tabei, Anal. Chem., 66, 285 (1994).

40. O. Niwa, T. Horiuchi, and H. Tabei, J. Electroanal. Chem., 367, 265 (1994).

41. N. Honda, M. Inaba, T. Katagiri, S. Shoji, H. Sato, T. Homma, T. Osaka, M. Saito, J. Mizuno, and Y. Wada, Biosens. Bioelect., 20, 2306 (2005).

42. T. Ito, N. Aoki, S. Kaneko, and K. Suzuki, Electrochem., 80, 305 (2012).

43. S. Kyung Kim, P. J. Hesketh, C. Li, J. H. Thomas, H. B. Halsall, and W. R. Heineman, Biosens. Bioelect., 20, 887 (2004).

44. B. Jin, W. Qian, Z. Zhang, and H. Shi, J. Electroanal. Chem., 411, 29 (1996).

45. C. A. Paddon, M. Atobe, T. Fuchigami, P. He, P. Watts, S. J. Haswell, G. J. Pritchard, S. D. Bull, and F. Marken, J. Appl. Electrochem., 36, 617 (2006).

46. K. J. Aoki, C. Li, T. Nishiumi, and J. Chen, J. Electroanal. Chem., 695, 24 (2013).

47. C. Li, K. J. Aoki, T. Nishiumi, and J. Chen, Report Electrochem., 3, 7 (2013).

48. H. Tabei, M. Morita, O. Niwa, and T. Horiuchi, J. Electroanal. Chem., 334, 25 (1992).

49. H. Tabei, O. Niwa, T. Horiuchi, and M. Morita, Electrochemistry, 61, 820 (1993).

50. T. Gueshi, K. Tokuda, and H. Matsuda, J. Electroanal. Chem., 101, 29 (1979).

51. D. J. Griffiths, Introduction to Electrodynamics, Second edition, Prentice Hall, p. 113 (1989).

52. K. Aoki, Y. Hou, J. Chen, and T. Nishiumi, J. Electroanal. Chem., 689, 124 (2013).

53. H. Zhang, K. Aoki, J. Chen, T. Nishiumi, H. Toda, and E. Torita, Electroanalysis, 23, 947 (2011). 